\title{
Ecosystem engineering, environmental decay and environmental states of landscapes
}

\author{
Xavier Raynaud, Clive G. Jones and Sébastien Barot \\ X. Raynaud (xavier.raynaud@ens.fr), UPMC-Paris6 UMR CNRS 7618, Biogéochimie et Ecologie des Milieux Continentaux, 46 rue d'Ulm, \\ FR-75230 Paris Cedex 05, France. - S. Barot, UMR CNRS 7618, Biogéochimie et Ecologie des Milieux Continentaux, 46 rue d'Ulm, \\ FR-75230 Paris Cedex 05, France. - C. G. Jones, Cary Inst. of Ecosystem Studies, PO Box AB, 2801 Sharon Turnpike, Millbrook, \\ NY 12545-0129, USA.
}

\begin{abstract}
Although environmental modification by ecosystem engineers influences species distributions and abundances and ecological process rates, general determinants of the environmental states of engineered landscapes are not well understood. Here we develop a general, spatially implicit model of engineered landscapes that includes parameters driving engineer populations (demographics, environmental modification) and environmental decay. We show that average environmental states and heterogeneities of landscapes are the result of a balance between parameters determining engineering rates and decay rates that can be expressed as a net engineering ratio (NER). This ratio highlights the need to include environmental decay in ecosystem engineering studies. Moreover, it defines a significant engineer as one that can alter the environment despite decay and generates expectations for different kinds of effects on the engineer, other species and ecological processes depending on ratio values. Finally, it suggests that, in general, decay places limits as to what can be inferred about engineer population dynamics from environmental dynamics and vice versa.
\end{abstract}

Environmental heterogeneity is a primary determinant of species distributions and abundances, and rates of ecological processes (Hutchings et al. 2000). Because all freeliving organisms alter their surrounding environments to some degree, they also generate environmental heterogeneity (Pickett et al. 2000, Wilson 2000). Ecosystem engineering (Jones et al. 1994, 1997) - alteration of abiotic resources and conditions by organisms independent of their assimilatory/dissimilatory (i.e. trophic) influences - is a very common type of environmental modification encompassing the activities of beaver, burrowing animals, molluscs, microbial crusts, desert shrubs and a great many other species (Darwin 1881, Johnston and Naiman 1990, Meadows and Meadows 1991, Gutiérrez et al. 2003, Wright et al. 2006, Lavelle et al. 2007). Engineering organisms affect communities and ecological processes at the patch level (Wright and Jones 2004), but also have landscape-level influence (Jones et al. 1997, Flecker 1997, Crooks 2002, Wright et al. 2002, Gutiérrez and Jones 2006, Hastings et al. 2007, Shachak et al. 2008). Despite this recognition and a growing number of studies examining landscape effects of engineers (Castilla et al. 2004, Badano et al. 2006, Bangert and Slobodchikoff 2006, Borthagaray and Carranza 2007), the parameters that generally determine the average environmental states and environmental heterogeneities of engineered landscapes are not well understood.
General models of ecosystem engineering (Gurney and Lawton 1996, Cuddington and Hastings 2004, 2008, Cuddington et al. 2009) have mostly focused on understanding the population dynamics of engineers, not environmental dynamics. These models do include environmental modification and feedbacks to the engineer, and, in some formulations, environmental states are permitted to decay following organismal abandonment or death (Gurney and Lawton 1996, Cuddington et al. 2009). Nevertheless, a focus on engineer population dynamics only informs environmental dynamics to the extent that it mirrors it; an aspect that, to our knowledge, has not been explicitly evaluated in general models. Other, species-specific models have explored some ramifications for environmental dynamics (Gilad et al. 2004, Wright et al. 2004, Barot et al. 2007, Blanchart et al. 2009, Wright 2009). Such speciesspecific models use a limited set of possible parameter values and therefore cannot inform the more general question of what drives the environmental state of landscapes across all kinds of engineer/environment combinations.

Here we present a general, spatially implicit model of engineered landscapes that includes parameters driving engineer population size - per capita engineering activity, environmental state-dependent population growth - and the dynamics of the environment - engineer modification, environmental decay. We conducted a sensitivity analysis 
on all model parameters to address two questions. 1) What determines average environmental states and environmental heterogeneities of landscapes? 2) How do these two features relate to engineer population dynamics? We use findings from the model to help define what might constitute a significant engineer with regard to environmental change, and accordingly what may need to be measured to ascertain this; and explore some general consequences for ecological processes, other species, and engineers.

\section{Model development}

Our model considers an initially unmodified landscape in which local populations of an ecosystem engineer can progressively modify the environmental state of locales they occupy. The landscape is assumed to be closed to immigration and emigration by the engineer. The model considers only those parts of a landscape that can be organismally modified; locales that cannot be engineered are excluded. A schematic of the full model is shown in Fig. 1, with parameters summarized in Table 1.

In the model, modifications are described as progressive environmental states $\left(E_{i}\right)$ represented as proportions of a landscape. This allowed us to generate a near-continuous description of environmental modification appropriate to many kinds of engineering organisms, and a discrete state description appropriate to yet other kinds. Many organisms can be viewed as affecting the environment by progressively modifying continuous variables. For example, tussock-forming Spartina alterniflora trap sediments, which accumulate. The accumulation progressively increases elevation, reducing plant stress from tidal inundation (Bouma et al. 2007, Van Hulzen et al. 2007). When a tussock dies, the sediment mound is progressively eroded by tides. Similarly, mound-building termites move soil when building nests, progressively altering soil characteristics such as bulk density and water retention capacity (Dangerfield et al. 1998). When termitaria are abandoned, mounds are progressively destroyed by weathering and other organisms, with concomitant reversion of soil characteristics. Other engineering organisms can be viewed as creating discrete states, even if modification toward a particular state is progressive. For example, Amazonian peccary species, Tayassuidae, create wallows, converting a patch of forest floor into one with rain filled depressions that, when abandoned, eventually revert to forest floor due to evaporation, and sediment and organic matter accumulation (Beck et al. 2010). Similarly, steep, bare slopes become stabilized by vegetation; revert to unstable soil following landslips; and become re-stabilized by vegetation (Reinhardt et al. 2010). Our model is, therefore, applicable to any circumstance where environmental modification by organisms is reversible by environmental decay along an approximately similar trajectory. Environmental modification that is cyclic (e.g. beaver converting a riparian forest to a clear cut with a pond; to a wetland following dam abandonment; that, if not re-engineered, reverts back to riparian forest; Wright et al. 2004) would require a modification of the model that connects fully modified states (such as wetlands) directly back to unmodified states (such as riparian forests).

Applying our general model to a specific system would require deciding whether environmental modifications caused by and affecting an engineer population are best described as discrete or continuous. Discrete states can be directly mapped onto model states (e.g. forest floor or peccary wallow; unstable slope or vegetation-stabilized slope). Continuous states require the environmental parameter(s) to be appropriately discretized (e.g. sediment elevation for Spartina categorized as elevation intervals that have detectably different effects on engineer population growth).

In the following sections, we develop and analyse the behaviour of models of increasing complexity in order to derive the simplest model that encompasses all essential features of ecosystem engineering by organisms and environmental decay. Although we evaluated the effects of random and environmental-state-dependent engineer movement within the landscape on model behaviour, movement did not qualitatively affect outcomes ('Sensitivity analyses' and Supplementary material Appendix A1). For reasons of analytical tractability and ease of interpreting simulations, we therefore exclude engineer movement within the landscape from our discussion of model development and the analyses. For all models, by definition $\Sigma E_{i}=1$, and $E_{1}$ represents the proportion of the unmodified state and $E_{n}$ the proportion of the most modified state. The models have two sets of equations. The first set describes the fate of the different states of the environment $\left(E_{i}\right)$, and the other set describes the fate of the population size of engineers in each state of the environment $\left(N_{i}\right)$. Model outputs focus on the steady state distribution of environmental states in landscapes, i.e. average (mean) and heterogeneity (variance). In the following, we first analyse the model where a fixedsize population of engineers transforms the landscape into

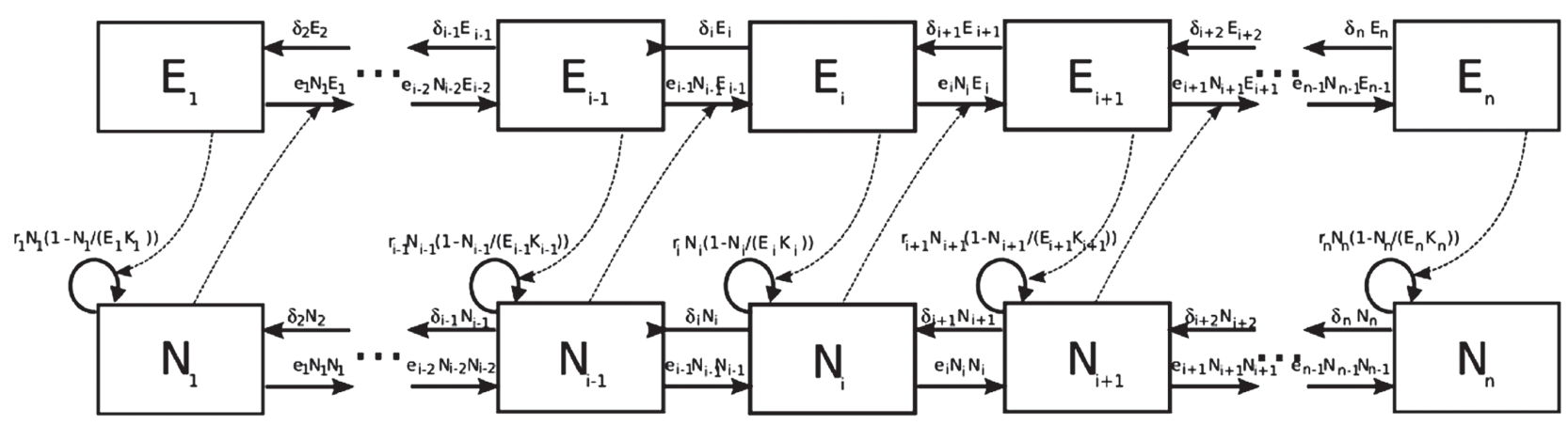

Figure 1. Schematic representation of the model. Arrows and parameters are explained in the text and Table 1. 
Table 1. Model parameters. In the text, symbols denoted by $*$ refer to steady state values and a bar above a symbol indicates its average value across all states.

\begin{tabular}{ll}
\hline Symbol & \multicolumn{1}{c}{ Parameter description } \\
\hline$n$ & number of environmental states \\
$E_{i}$ & proportion of landscape in environmental state $i$ \\
$N_{i}$ & engineer population size in environmental state $i$ \\
$\delta_{i}$ & environmental decay rate from state $i+1$ to state $i$ \\
$e_{i}$ & per capita engineering activity of engineers between state \\
& $\quad i$ and $i+1$ \\
$r_{i}$ & intrinsic population growth rate of engineers in state $i$ \\
$K_{i}$ & carrying capacity of state $i$ \\
$N_{\text {tot }}$ & total engineer population size in landscape. $N_{\text {tot }}=\sum_{i=1}^{n} N_{i}$ \\
NER & net engineering ratio. NER $=\bar{K} e /(n \delta)$ \\
\hline
\end{tabular}

several environmental states; then add a population dynamic model of the engineer.

\section{Landscape modification}

In the absence of reproduction, mortality, and landscapelevel immigration and emigration, engineers will modify the environment as a function of their per capita engineering activity and population size, creating up to $n$ environmental states. Each environmental state with a proportion greater than zero can be occupied by engineers, expressed as their population size in these states $\left(N_{1}, \ldots, N_{n}\right)$. Engineer population dynamics in each state are based on the hypothesis that changes in size, $N_{i}$, are proportional to changes in environmental states, $E_{i}$ (i.e. when a proportion of the landscape is converted from state $i$ to state $i+1$, the same proportion of organisms shifts from state $i$ to state $i+1)$. All engineered environmental states $(i=2, \ldots, n)$ have an intrinsic tendency to decay to the previous, less modified, state. This yields the following set of equations characterising the dynamics of all environmental states $(i=1, \ldots, n)$ and engineer population sizes therein:

$\frac{d E_{i}}{d t}=-\delta_{i} E_{i}+\delta_{i+1} E_{i+1}+\tau_{i-1} E_{i-1}-\tau_{i} E_{i}$

$\frac{d N_{i}}{d t}=-\delta_{i} N_{i}+\delta_{i+1} N_{i+1}+\tau_{i-1} N_{i-1}-\tau_{i} N_{i}$

where $\delta_{i}$ is the environmental decay rate of state $i$ (i.e. the rate at which a state is converted from state $i$ to $i-1$ ), and $\tau_{i}$ is the engineering rate in state $i$ (i.e. the rate at which a state is converted from state $i$ to $i+1)$. $\delta_{i}$ represents the rate at which physical and chemical forces (e.g. structural collapse due to gravity; erosion by wind, rain, tides or runoff; oxygenation reversal of low redox effects; etc.), or biological forces (e.g. microbial degradation; succession following abandonment; etc.) reduce the degree of environmental modification created by the local engineer population. We assume that the engineering rate, $\tau_{i}$, is a function of the population size in that state, $N_{i}$, and the per capita engineering activity in state $i, e_{i}$. The latter represents the intrinsic capacity of an individual engineer to non-trophically modify the environmental state via the creation and maintenance of physical structures and chemical conditions. We assume that this per capita engineering activity $\left(e_{i}\right)$ is independent of population density so that engineering is solely a reflection of population size. Hence $\tau_{i}=e_{i} N_{i}$. Decay rate $\left(\delta_{i}\right)$ and per capita engineering activity $\left(e_{i}\right)$ parameter values can be different between states. We set the decay rate of the first state $\delta_{1}$ to 0 because no decay occurs in the first unmodified state. Also, because we consider a finite number of environmental states, $n$, a right boundary condition exists, with $\delta_{n+1}=0$. Similarly, there is a right boundary condition for engineering, $e_{n}=0$ (i.e. the $n$th state is the most modified environmental state possible), and we set $e_{0}=0$ as the 0 th state does not exist.

In the above formulation, the population size in state $i$ at steady-state $\left(N_{i}^{*}\right)$ can be obtained as a function of population size in state $i-1$ by setting the sum of all $d N_{j} / d t$ to 0 where the sum runs from $j=1$ to $i-1$ :

$N_{i}^{*}=\frac{e_{i-1}}{\delta_{i}}\left(N_{i-1}^{*}\right)^{2}$

There is a trivial equilibrium $N_{i}^{*}=0$ for all $i$ given by the initial condition $N_{i}=0$ for all $i$. Otherwise, if initially $N_{i} \neq 0$ for at least one $i$, then at steady state, all environmental states can be occupied by engineers depending on the ratio $e_{i-1} / \delta_{i}$. In this case, and because changes in engineer population sizes are proportional to changes in population densities, the proportions of the different environmental states are also proportional to the population sizes of engineers in the corresponding state. Note also that in the absence of reproduction, mortality, and immigration/ emigration, the total population in the landscape $N_{t o t}$, is constant over time and equal to the initial population size (i.e. engineers are only shifted from one environmental state to another). Here then, a condition that allows the engineers to be uniformly distributed across all the states at equilibrium (e.g. for which $N_{i}^{*}=N_{t o t} / n$ for all $i$ ) will also lead to all environmental states being present in the same proportion (maximum landscape environmental heterogeneity and evenness). This condition is:

$\frac{N_{t o t}}{n} \frac{e_{i-1}}{\delta_{i}}=1$

If this condition is not met, then the distribution of population sizes and proportions of environmental states will depend on the value of the ratio $N_{t o t} e_{i-1} /\left(n \delta_{i}\right)$. First, suppose that all $e_{i}$ and all $\delta_{i}$ are equal between states $\left(e_{i}=e\right.$ and $\left.\delta_{i}=\delta\right)$. If $N_{\text {tot }} e /(n \delta)<1$, the environmental decay rate is too high for organisms to convert the landscape toward more modified states, and the landscape is mostly comprised of less modified states. In contrast, if $N_{t o t} e /(n \delta)>$ 1 , organisms are able to convert most of the landscape to more modified states. In both cases, $N_{i}^{*}$ or $E_{i}^{*}$ are ordered monotonically and increase or decrease with increasing values of $i$ depending on the ratio $e / \delta\left(N_{1}^{*}>N_{2}{ }^{*}>\ldots\right.$ $>N_{n-1}{ }^{*}>N_{n}^{*}$ when $N_{\text {tot }} e /(n \delta)<1$, or $N_{1}^{*}<N_{2}^{*}<\ldots$ $<N_{n-1}{ }^{*}<N_{n}{ }^{*}$ when $\left.N_{t o t} e /(n \delta)>1\right)$. Similar conclusions hold when $e_{i}$ and $\delta_{i}$ are different between states under the condition that Eq. 4 is kept true for all $i$. If, however, for a particular state $N_{\text {tot }} e_{i-1} /\left(n \delta_{i}\right)<1$, the environmental decay rate is too high for organisms to further modify this state, and there is a tendency to return the $i-1$ th state; whereas if $N_{t o t} e_{i-1} /\left(n \delta_{i}\right)>1$, organisms will transform most of the $i-1$ th state of the environment towards the $i$ th state. 


\section{Population dynamics}

Our objective here is to modify the simplest model (i.e. Eq. 1, 2) to include population growth of engineers. We assume this follows a logistic equation whose parameters depend on environmental state. For each environmental state, engineer population growth is:

$\frac{d N_{i}}{d t}=r_{i} N_{i}\left(1-\frac{N_{i}}{E_{i} K_{i}}\right)$

where $r_{i}$ represents the intrinsic population growth rate of engineers in state $i$, and $K_{i}$ is the carrying capacity of state $i$ if state $i$ occupies the entire landscape. Similarly to $\delta_{i}$ and $e_{i}$, parameters values for $r_{i}$ and $K_{i}$ can be different between states. Because the entire landscape is not necessarily in the same state, the carrying capacity of this state also depends on its proportion in the landscape. Since the landscape is closed to immigration and emigration, $r_{i}$ in this Eq. 5 is the result of only birth and death. Replacing $\tau_{i}$ by $e_{i} N_{i}$ in Eq. 1 and 2 and coupling Eq. 5 with Eq. 2 yields the equations for the full model:

$$
\begin{aligned}
\frac{d E_{i}}{d t}= & -\delta_{i} E_{i}+\delta_{i+1} E_{i+1}+e_{i-1} N_{i-1} E_{i-1}-e_{i} N_{i} E_{i} \\
\frac{d N_{i}}{d t}= & -\delta_{i} N_{i}+\delta_{i+1} N_{i+1}+e_{i-1} N_{i-1} N_{i-1} \\
& -e_{i} N_{i} N_{i}+r_{i} N_{i}\left(1-\frac{N_{i}}{E_{i} K_{i}}\right)
\end{aligned}
$$

Let us first assume that parameters $\delta_{i}, e_{i}, r_{i}$ and $K_{i}$ are equal between states $\left(\delta_{i}=\delta, e_{i}=e, r_{i}=r\right.$ and $\left.K_{i}=K\right)$. In this case and at steady state, population size in state $i$ has an equilibrium value $E_{i} K$ and the population size in state $i$ is related to the total population size and the proportion of the landscape in state $i N_{i}^{*}=N_{t o t}^{*} E_{i}^{*}$. For the same reasons, the total population $N_{\text {tot }}$ follows the logistic growth equation:

$\frac{d N_{t o t}}{d t}=r N_{t o t}\left(1-\frac{N_{t o t}}{K}\right)$

If engineers are present in state $i$ (e.g. $N_{i}>0$ ) and $r>0$, then $N_{\text {tot }}^{*}=K$ and $N_{i}^{*}=K E_{i}^{*}$ at steady state. In this case, all environmental states will be in equal proportion at steady state under the condition $K e /(n \delta)=1$. If $K e /(n \delta)<1$, environmental decay has a greater influence than engineering and the landscape will be mostly occupied by unmodified or less modified states; whereas if $\operatorname{Ke} /(n \delta)>1$, states that are more modified occupy a greater proportion of the landscape than unmodified or less modified states. Similar conclusions hold when parameters $\delta_{i}$ and $e_{i}$ differ between states under the condition that

$$
\frac{K}{n} \frac{e_{i-1}}{\delta_{i}}=1
$$

is kept true. If $K_{i}$ differs between states, this condition is insufficient to result in all states occurring in equal proportions because the equality $N_{i}^{*}=K_{i} E_{i}$ is not always true (Supplementary material Appendix A1 Fig. A1).

\section{Sensitivity analysis}

Our analysis of the final general model (Eq. 6 and 7) generates some insights into model behaviour for simple situations. Assuming all parameters are equal across states, the distributions of proportions among states only depends on the ratio $\mathrm{Ke} /(n \delta)$ (section 'Population dynamics'). When this ratio is above 1 , engineers are able to modify the landscape at a greater rate than it tends to return to its unmodified state; whereas when the ratio is less than 1 , decay prevents engineers from modifying the landscape and it remains mostly unmodified. It is likely, however, that each environmental state is characterized by particular engineering, decay or population growth rates. For example, it is possible that the more modified the environment, the more difficult further modification becomes (e.g. $e_{i}<e_{i-1}$ ), and the more rapidly it tends to return to a less modified state (e.g. $\delta_{i}>\delta_{i-1}$ ). Many other parameter combinations might exist across engineer species and landscapes.

To better understand the consequences of differences in parameter values among states (e.g. $r_{i} \neq r_{j}$ ), we conducted a global sensitivity analysis (model implemented in $\mathrm{C}++$; available from the authors) to ascertain how outputs depend on inputs (Saltelli et al. 1999). Repeated model simulations drew parameter values from uniform distributions for a numerical model parameterised for 5 environmental states. We ensured that results were qualitatively independent of the number of states by running simulations with more (up to 8 ) or less (down to 2) states (Supplementary material Appendix A1 Fig. A2). In order to have a broad yet precise understanding of the behaviour of the 5 state model, our analyses compared 4 different sets of 10000 simulation runs with different assumptions about the relationships between parameter values among states (40000 simulations in total). In all simulation sets, the model was initialized with a landscape comprised of only the unmodified state and a population size of engineers with values uniformly drawn from 1 to 10 . In the first simulation set, hereafter termed the full random parameter set, FR, we made no a priori assumptions about relationships between parameter values among states; all parameter values for every state were drawn independently from their respective ranges. Values for $\delta_{i}$ were assumed to be uniformly distributed from 0 to $50 ; e_{i}$, and $r_{i}$ from 0 to 10 ; and $K_{i}$ from 1 to 49. Distribution ranges for the different parameters were chosen so as to obtain all possible combinations of states in the model (i.e. simulations could lead to a predominance of more modified or less modified environmental states). The FR set parameter distributions are unlikely to be realized for many engineer species but nevertheless cover the full range of possible variation in inputs. As a consequence, any emergent properties apparent in the outputs are due solely to model structure and not parameterisation. In the second set, hereafter called the average random parameter set, AR, parameter values were fixed to the respective mean values found over all states in the FR set (e.g. $r_{i}=$ $\left.r_{j}=\bar{r}\right)$. Because parameter values did not change between states in this set, it had the general properties described earlier (i.e. state distribution is determined solely by $\mathrm{Kel} n \delta$ ). Moreover, since this set was based on average parameter values of the FR set, the comparison between $A R$ and FR 
revealed how model behaviour was affected by variation in parameter values among states. Finally, we used two additional parameter sets in which carrying capacities were negatively (negative feedback parameter set, NF) or positively (positive feedback parameter set, PF) correlated with environmental states. In these two sets, all parameters were chosen to be in the same range as those in the FR and AR sets, while ensuring that carrying capacities $\left(K_{i}\right)$ respectively decreased or increased with increasing $i$.

Model outputs of environmental states in the landscape were characterised by two indices describing landscape environmental heterogeneity and landscape average state. Landscape environmental heterogeneity was derived from the number of states with a proportion greater than 0 and their relative proportions. We used an index identical to Shannon evenness $J^{\prime}$ to estimate this heterogeneity, calculated as $-\Sigma E_{i} \log \left(E_{i}\right) / \log (n)$. This index ranged from 0 to 1 ; where 0 corresponded to a homogeneous landscape (only one state is present), and 1 corresponded to a landscape with maximum heterogeneity with all states equally represented in proportion $1 / n$. Because landscapes that are fully engineered or completely unmodified both have low values for the heterogeneity index, we also calculated the landscape average state as $\bar{E}=\Sigma_{i} i E_{i}$ to distinguish these two situations.

As pointed out earlier, our model does not explicitly include engineer movement within the landscape. To evaluate whether or not this influenced findings, we examined the qualitative effect of movement using simulations with a modified version of the general model. The general behaviour of the model was not affected by random or environmental state-dependent movement, even when a relatively large proportion of organisms were allowed to move among states (up to $25 \%$ per time step; Supplementary material Appendix A1 and Fig. A3). As a consequence, we ignore movement in our subsequent analyses, results and discussion.

\section{Results}

\section{Dynamics of environmental states and engineer populations}

Figure 2 illustrates temporal dynamics of population sizes and environmental states in a landscape with parameter values $\bar{e}=1.2, \bar{\delta}=5, \bar{K}=50$ and $\bar{r}=1.1$. When initiated with one environmental state occupied by a small number of engineers, the total population grows toward the landscape equilibrium value, $K$. At the same time, the landscape is progressively transformed, reaching an equilibrium value for each environmental state.

\section{Environmental states in the sensitivity analyses}

In the FR set, the range of proportions of each state was roughly similar. Across the 10000 simulations with this set, proportions of each state varied from 0 to $80 \%$ of the landscape, indicating that nearly all possible combinations of environmental state distributions occurred in the sensitivity analysis. The simulations encompassed situations where all of the landscape remained unmodified, to
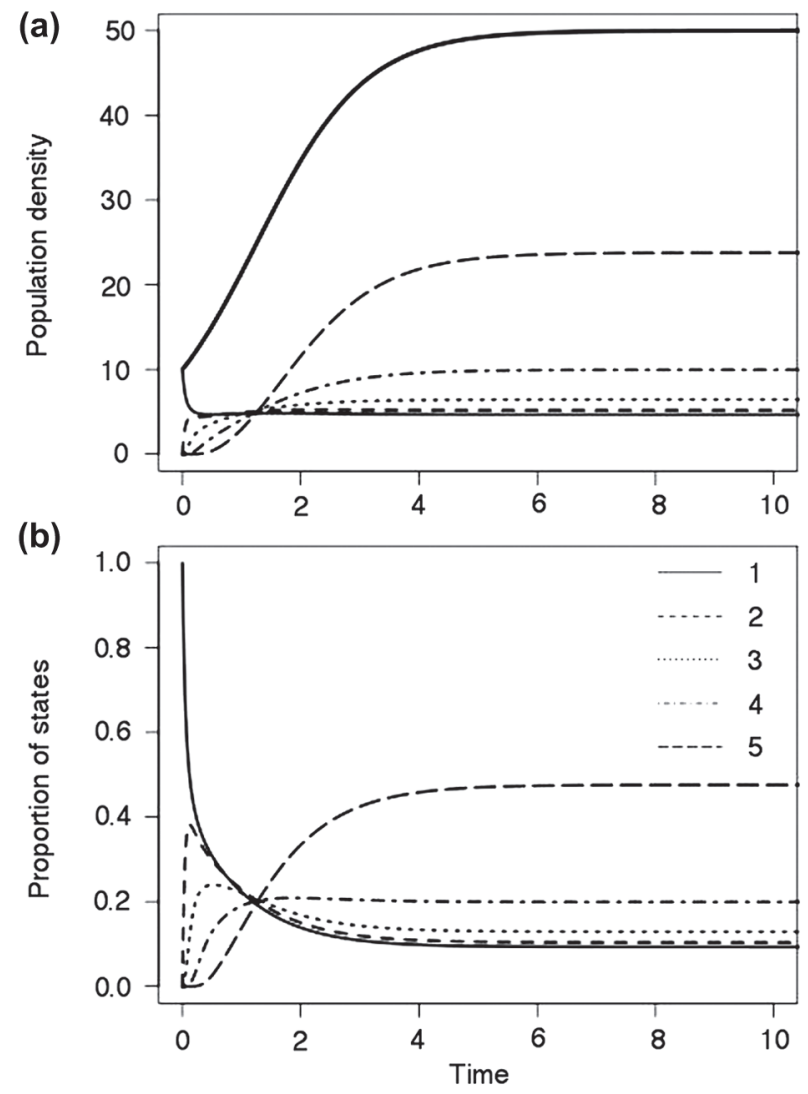

Figure 2. (a) Engineer population size, and (b) Proportion of environmental states in the landscape over time. The thick line in (a) is the total engineer population size in the landscape, $N_{t o t}$. Thin lines are population sizes (a) and proportions (b) for 5 different environmental states (legend, 1-5). In this simulation, all parameters were set to equality among states with $\bar{e}=1.2, \bar{\delta}=5, \bar{K}=50$ and $\bar{r}=1.1$.

situations where most of the landscape was fully modified. The distribution of states in the NF set was similar to that observed in the FR set. In contrast, the positive feedback set $(\mathrm{PF})$ was associated with a lower proportion of intermediate states; the environment was either mostly unmodified or substantially modified (Supplementary material Appendix A1 Fig. A4).

\section{Steady state population size and model parameters}

Under the logistic growth equation (Eq. 5), the theoretically expected population size in one state is $E_{i} K_{i}$. In simulations using the FR set, there was a strong linear relationship between realized population sizes in each state and theoretical expectations $\left(N_{i}^{*} \sim E_{i}^{*} K_{i} ; R^{2}>0.55\right.$ for all states; Supplementary material Appendix A1 Fig. A1). Comparing the total population size in the landscape at steady state to the theoretical total population size $\left(N_{t o t}^{*}=\right.$ $\left.\Sigma E_{i}^{*} K_{i}\right)$ also yielded a strong linear relationship $\left(R^{2}=0.73\right.$; Supplementary material Appendix A1 Fig. A1), but revealed cases where the realized population size was greater or less than expected. Situations where total population size exceeded expected values occurred when environmental states with high carrying capacities were present in high proportions; whereas cases where total population size was 
less than expected occurred when most of the landscape was comprised of states with low carrying capacities.

\section{Population versus environmental states}

Total population size in the landscape was largely determined by population growth parameters in each state. In simulations with the FR set, there was a positive relationship between the total population size in the landscape and the average state of the landscape $\left(R^{2}=0.27\right.$, Fig. 3a); environmental states affected population growth and vice versa, but there was imperfect correspondence reflected in the low $R^{2}$. The low $R^{2}$ was not due to differences in parameter values between states, because the AR set - where values for a parameter were identical between states showed a similarly weak relationship $\left(R^{2}=0.25\right.$; data not shown). The relatively weak inter-relationships were due to the influence of environmental decay rates whose parameter values were independent of population size.

Feedbacks also showed the influence of decay, albeit differentially. Negative feedbacks (where carrying capacities of environmental states were constrained so that they were negatively correlated with the rank of the environmental state) showed a negative weak relationship between total population size and average environmental state (Fig. 3b; $\left.R^{2}=0.08\right)$. When decay rates were low, organisms could modify the environment. Nevertheless, because such environments were associated with low carrying capacities, the total population size was low. When decay rates were high, relatively unmodified states were associated with high carrying capacities, and, hence, high total population sizes. In contrast, positive feedbacks (where carrying capacities of environmental states were constrained so that they were positively correlated with the rank of the environmental state) led to a strong, positive relationship with the highest $R^{2}$ found across sets (Fig. 3c; $R^{2}=0.76$ ); nevertheless, the influence of decay was still apparent from the unexplained variance.

\section{Model parameters and the landscape}

Model analyses indicated that the average states and heterogeneities of landscapes were controlled by $\mathrm{Ke}_{i-1} /\left(n \delta_{i}\right)$. When $K e_{i-1} /\left(n \delta_{i}\right)=1$, all environmental states were equally represented such that the landscape average state was $\bar{E}=3$. Because all parameters were randomly chosen for the FR set, equality could not be directly verified. However, using average values of $r_{i}, K_{i}, e_{i}$ and $\delta_{i}$ across environmental states, an average ratio, NER $=\bar{K} \bar{e} /(n \bar{\delta})$, could be estimated. As expected, this ratio - which we define as the net engineering ratio (NER) - was correlated to the average states of landscapes (Fig. 4a) and their heterogeneities (Fig. 4b). When NER $=1$, the average states of landscapes approached the intermediate environmental state, and landscapes had maximum environmental heterogeneities (note, however, that when the landscape average state equalled the intermediate state this did not mean that all states were necessarily equally represented). When NER was $\neq 1$, landscape heterogeneity was reduced; unmodified states dominated when NER $<1$, while highly modified states dominated when NER $>1$. In the AR set, where all parameters were equal among states, the relationships between NER and the average environmental states and environmental heterogeneities of landscapes were curvilinear (Fig. 4, grey curves). Positive or negative feedbacks did not markedly change these relationships (Supplementary material Appendix A1 Fig. A5), although positive feedbacks were associated with a lower evenness of states compared to situations with negative feedbacks or no feedbacks (Supplementary material Appendix A1 Fig. A5b, A5d).

\section{Discussion}

Our model is a simplification. It omits immigration and emigration from the landscape. Although we showed that movement within the landscape had no obvious qualitative influence (Supplementary material Appendix A1 Fig. A3), we did not explicitly evaluate its quantitative effects, and did not include movement in other analyses. The model ignores the 'unmodified matrix' of a real landscape (i.e. not all organisms can occupy and modify all the landscape: the habitat may be unsuitable; it may be suitable but not modified, such as beaver living in geomorphic ponds that do not build dams; it may be suitable, but local conditions prevent modification, such as soil too hard to dig). Our analysis of NER was conducted at steady state, although the outcomes can also be thought of as progressive tendencies
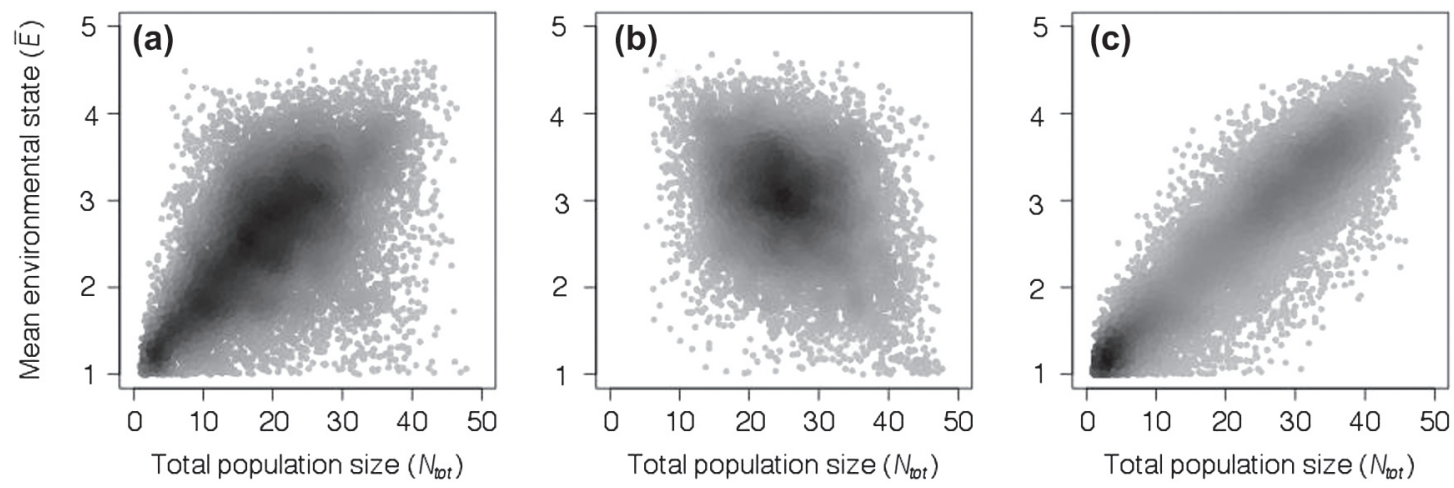

Figure 3. Engineer total landscape population size vs mean landscape environmental state for the case of 5 states. (a) Full random parameterization set (FR); (b) negative engineering feedback set (NF); and (c) positive engineering feedback set (PF). Each point in each graph is the outcome of one simulation. Shading indicates point density. 

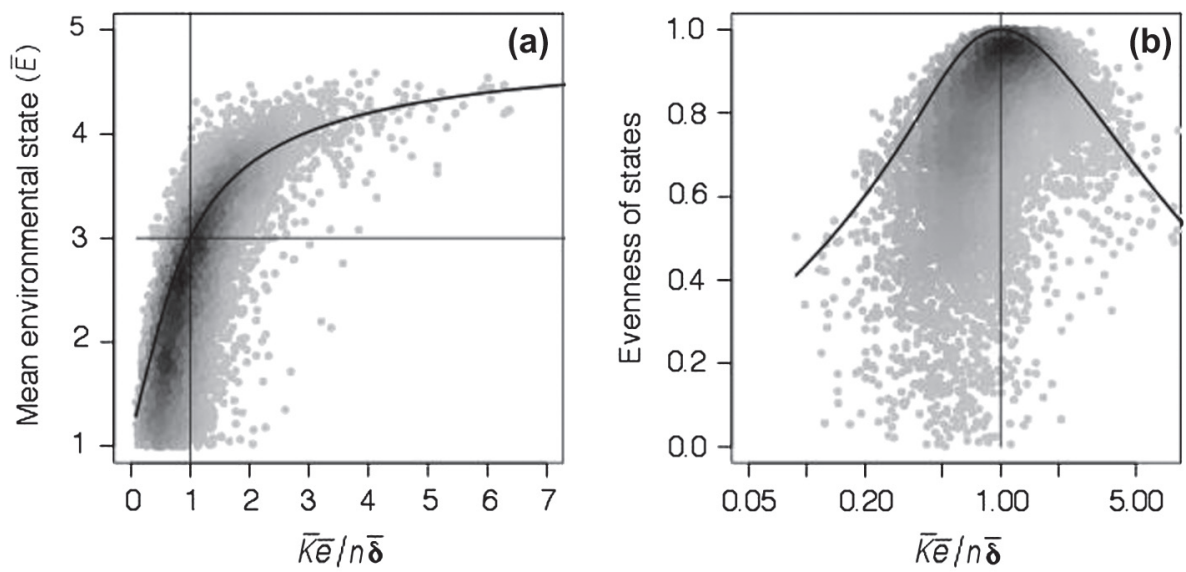

Figure 4. (a) Relationship between mean environmental state of landscapes and the net engineering ratio (NER) for the case of 5 states. Thin vertical and horizontal lines show where NER $=1$ and the average state is equal to the intermediate state $\bar{E}=(n+1) / 2$; (b) Relationship between landscape environmental heterogeneities and NER. Note the logarithmic scale of the $x$-axis. Each point in each graph is the outcome of one simulation. Shading indicates point density.

over time. We treated unmodified to fully modified patches as a linear bidirectional sequence, ignoring the possibility of alternate environmental states, irreversible transitions, and cyclic behaviour. Inclusion of such features would very likely affect quantitative patterns, and the degree to which they might alter our qualitative conclusions remains to be seen. Adapting the spatially implicit model or developing a spatial explicit version would no doubt clarify their influences. Nevertheless, the current model indicates the fundamental importance of including environmental decay to understand and predict the distribution of environmental states of engineered landscapes - the issue on which we focus our discussion.

The relevance of the net engineering ratio $(\mathrm{NER}=$ $\bar{K} \bar{e} /(n \bar{\delta}))$ that emerges from our analyses of model output across all possible parameter combinations makes intuitive sense. Average environmental states and heterogeneities of landscapes result from the balance of two forces: the 'push' of the engineer and the 'pull' of decay. The push (i.e. $\bar{K} \bar{e}$ ) is the rate at which organisms are able to convert unmodified patches into progressively more modified states, and is a function of their per capita engineering activities, intrinsic population growth parameters and engineering feedbacks $(+,-$ or 0$)$. The pull (i.e. $n \bar{\delta})$ is the intrinsic rate with which patches continuously revert to less modified states, and arises from physical, chemical and other biological forces. This finding has some interesting and potentially important general ramifications. The example of Spartina (see 'Model development') illustrates these two forces. The push is related to tussock growth and stem density which attenuate hydrodynamic forces causing sediment deposition in the tussock vicinity, and increased elevation at the marsh scale. The increase in elevation reduces plant growth constraints, a positive engineering feedback (Van Hulzen et al. 2007). The pull is the result of hydrodynamic forces that remove sediment from the marsh. Marsh elevation depends on the relative magnitude of the two opposing forces. If sediment accretion due to rapid plant growth at high tussock densities exceeds tidal removal of sediment, then Spartina will transform the marsh; whereas this will not occur if sediment removal by tides exceeds sediment trapping by Spartina.

The model output reveals three general steady state situations with respect to NER and the average environmental states and heterogeneities of landscapes. When NER $>1$, engineering rates are greater than decay rates and organisms both transform and homogenize the environmental landscape despite decay. When NER $\approx 1$, engineering and decay rates result in intermediate average environmental states of landscapes with high environmental heterogeneities. When NER $<1$, decay homogenizes the environment despite the engineering, and organisms leave little trace in landscapes. It follows that: 1) organisms causing apparently superficially large or trivial engineering effects on the environment could, at steady state, or eventually over time, have conversely small or large landscape effects on environmental states depending on decay rates; 2) different engineer rate/decay rate combinations can have the same NER (e.g. high/high and low/low are equivalent); 3) since NER depends on both organism and environment, the same species in two landscapes with different decay rates, or two species with different engineering rates in a landscape where the decay rate is the same, can result in a different NER. Thus the significance of the ecosystem engineering in terms of the degree of control exerted on the distribution of environmental states in a landscape by a species cannot be ascertained without consideration of environmental decay. In the context of this model, a significant ecosystem engineer can be defined as one that can alter the distribution of environmental states in the face of decay. So here, ecosystem engineering emerges as being fundamentally context-dependent, reflecting the more general observation that unless an organism can exert perfect control over its abiotic environment - likely it never can - its effects must necessarily be somewhat environmentally context-dependent (Jones and Gutiérrez 2007).

Engineered landscapes in the real world may never be at or even approach steady state. Nevertheless, there are situations, particularly at the extremes of push versus pull, where the consequences of engineering on landscape 
environmental heterogeneity might be inferred. Each bubble net made by one or more humpback whales for fishing (Sharpe 1984) is very ephemeral. So unless there are very large numbers of whales continuously making bubble nets in marine landscapes, such environmental modification is clearly transient, significant as it may be to the whales and their prey caught in the nets. Unless the pyramids of Egypt get repaired they will disappear, despite the burst of human engineering activity a few thousand years ago. As long as a forest is present it will continue to modify the understory microclimate, but if cut down the microclimate will rapidly decay to ambient. Termite mounds that are continuously maintained, or abandoned and later reoccupied, will leave a lasting, heterogeneous imprint on the landscape as long as termites are present; abandoned mounds in very arid environments with little or no wind and no termites will last a long time, but eventually disappear (Moore and Picker 1991).

There are, however, many other engineered landscapes where it may be much harder to infer whether push, pull, or both are controlling environmental state distributions For example, when bears periodically dig in alpine meadows (Butler 1992), is it bears, mound erosion and pit infill, or both, that determine the environmental state? What is the NER of burrowing worms in tidal mudflats (Volkenborn et al. 2007)? There is a high engineering rate, but each tide could erase the traces. In these and perhaps many other systems where push and pull rates may not markedly differ, and could even be the same, drawing conclusions about the determinants of the environmental state cannot be made without recourse to measures of both engineering rates and decay rates. Nevertheless, it would appear that empirical studies of ecosystem engineering have paid far less attention to decay than to engineering (Strayer and Malcom 2007), despite awareness of engineering legacies (Hastings et al. 2007). Our model suggests that a much more equal treatment is needed. The NER indicates the kinds of parameters and interrelationships that may be needed to understand engineered environment dynamics.

We showed correlations between the landscape average state and population size in the landscape, as one might expect (Fig. 3a). Nevertheless, across parameter space these relationships accounted for relatively small amounts of the total variance. While some of the unexplained variation was due to the variety of values for demographic parameters used in our general model, it was also clear that decay played a major role, although we made no attempt to partition these two sources of variation. As pointed out in the introduction, one justification for our inclusion of environmental decay was to examine the degree to which environmental dynamics mirrors population dynamics. Clearly it does not, and this is due to environmental decay. As a result, even though population dynamics affects environmental dynamics and vice versa, environmental dynamics is not the simple inverse of the population dynamics of the engineer. Our results indicate that knowledge of the parameters driving engineer population dynamics may be insufficient to understand environmental dynamics; environmental decay rates must also be known.

Our analyses also showed that engineering feedbacks (increasing or decreasing $K_{i}$ ) can influence the landscape average state, also as one might expect. Nevertheless, a wide range of landscape average states was possible with both negative and positive feedbacks, with substantial overlap (Supplementary material Appendix A1 Fig. A5a, A5c). Both negative and positive feedbacks also generated a similar, very broad range of values for landscape environmental heterogeneity, although positive feedbacks were associated with lower values (Supplementary material Appendix A1 Fig. A5b, A5d). This occurred because when decay rates were sufficiently high, organisms could not modify the landscape rapidly enough; whereas low decay rates led to run-away transformation of the landscape due to increasing population sizes. As a result, with positive feedbacks, landscapes tended to be either relatively unmodified or highly modified; intermediate states were less common (Supplementary material Appendix A1 Fig. A4). Environmental decay and engineering feedbacks are both recognized as important determinants of population dynamics (Gurney and Lawton 1996, Cuddington et al. 2009). Our results indicate that both are also important for understanding environmental dynamics, despite the fact that population sizes and landscape states can be weakly correlated.

Despite model limitations, what do our findings suggest about the consequences of environmental decay for other affected species and ecological processes, and ecosystem engineers? For ecological processes and other species, the model generates expectations for different kinds of effects based on NER. When engineering rates markedly exceed decay rates $(\mathrm{NER} \gg \mathrm{P})$, the transformation and homogenization of the environmental states of a landscape should lead to relatively uniform ecological process rates under engineer control, and the elimination of species that cannot persist in the engineered environment with replacement by species that can. When engineering rates and decay rates are not that dissimilar $(\mathrm{NER} \approx 1)$, ecological process rates will be heterogeneous in the landscape (Gutiérrez and Jones 2006), and there will be diverse opportunities for species persistence encompassing those limited to modified habitat or unmodified habitat (Wright et al. 2002), and generalists capable of living in multiple environment types (Badano et al. 2006). When decay rates markedly exceed engineering rates (NER $<<1$ ), ecological process rates are controlled by the ambient environment with only transient influence by the engineer. Most species will be those that can persist in unmodified environments irrespective of degree of habitat specialization (i.e. both unmodified habitat specialists and habitat generalists). Species that can occupy engineered habitats, in particular engineered habitat specialists, must be capable of finding and exploiting those habitats during their transient existence, implying requirements for high dispersal capability, and rapid growth and reproduction.

The consequences of environmental decay for engineers are generally in accord with prior findings. The inclusion of environmental decay - particularly varying decay rates (cf. fixed, random or exponential decay; Gurney and Lawton 1996, Cuddington et al. 2009) - further broadens the already wide open possibilities for engineer population dynamics (Gurney and Lawton 1996, Cuddington et al. 2009), rather than constraining them. High decay rates relative to engineering rates reduced the influence of feedbacks from the engineered environmental state to 
population size. As previously pointed out (Cuddington et al. 2009), this suggests that high relative decay rates could prevent positive engineer feedbacks from resulting in run-away population growth, and negative feedbacks from resulting in population collapse. The decay rate of engineered environmental states may stabilize engineer dynamics in some circumstances.

Across all parameter space, landscape states were not strongly correlated to total population sizes. While feedbacks led to detectable differences in the relationship between average states and total population size, they did not markedly change these relationships, and had no clear effect on environmental heterogeneity. Thus despite the fact that environmental modification is what characterizes ecosystem engineering, there are limitations to what can be inferred about population dynamics from environmental dynamics, and of course vice versa, unless decay parameters are known. As with environmental dynamics, the NER indicates the kinds of parameters and interrelationships that may be needed to understand engineer population dynamics. Finally, we suggest that the above limitations to inference about engineer dynamics from environmental dynamics are relevant to considerations of engineer fitness. An engineering species could gain fitness benefits from positive feedbacks, or experience decreased fitness from negative feedbacks. However, both situations could occur with or without detectable changes in environmental states, depending on decay rates. Thus the distinction between niche construction (Laland et al. 1999, OdlingSmee et al. 2003) and niche change (Dawkins 2004) cannot be inferred from engineer-induced environmental change even when the engineering affects fitness.

Acknowledgements - The authors thank E. Thebault for constructive comments. XR thanks CNRS and UPMC-Paris 6, and SB thanks CNRS and IRD for financial support. CGJ thanks the following sources of financial support: The Cary Inst. of Ecosystem Studies; the state and region of the Ile de France (Chaire Internationale de Recherche Blaise Pascal, via the Fondation de l'École Normale Supérieure); le Ministère de l'Alimentation, de l'Agriculture et de la Pêche; AgroParisTech; and the Royal Netherlands Academy of Arts and Sciences. Work by CGJ was conducted while a visiting professor, UMR CNRS 7618, Biogéochimie et Ecologie des Milieux Continentaux, 46 rue d'Ulm, FR-75230 Paris CEDEX 05, France; invited professor, Direction Scientifique, AgroParisTech, 19 avenue du Maine, FR-75732 Paris CEDEX, 15 France; and a Royal Netherlands Academy for Arts and Sciences Visiting Professor, Centre for Estuarine and Marine Ecology, Netherlands Inst. of Ecology (Now the Royal Netherlands Inst. for Sea Research), Korringaweg 7, NL-4401 NT, Yerseke, The Netherlands. This paper is a contribution to the program of the Cary Inst. of Ecosystem Studies.

\section{References}

Badano, E. I. et al. 2006. Assessing impacts of ecosystem engineers on community organization: a general approach illustrated by effects of a high-Andean cushion plant. - Oikos 115: 369-385.

Bangert, R. K. and Slobodchikoff, C. N. 2006. Conservation of prairie dog ecosystem engineering may support arthropod beta and gamma diversity. - J. Arid. Environ. 67: 100-115.
Barot, S. et al. 2007. Self-organization in a simple consumerresource system, the example of earthworms. - Soil Biol. Biochem. 39: 2230-2240.

Beck, H. et al. 2010. Do neotropical peccary species (Tayassuidae) function as ecosystem engineers for anurans? - J. Tropic. Ecol. 26: 407-414.

Blanchart, E. et al. 2009. SWORM: an agent-based model to simulate the effect of earthworms on soil structure. - Eur. J. Soil Sci. 60: 13-21.

Borthagaray, A. I. and Carranza, A. 2007. Mussels as ecosystem engineers: their contribution to species richness in a rocky littoral community. - Acta Oecol. 31: 243-250.

Bouma, T. J. et al. 2007. Spatial flow and sedimentation patterns within patches of epibenthic structures: combining field, flume and modelling experiments. - Continental Shelf Res. 27: $1020-1045$.

Butler, D. R. 1992. The grizzly bear as an erosional agent in mountainous terrain. - Z. Geomorphol. 36: 179-189.

Castilla, J. C. et al. 2004. Marine ecosystem engineering by the alien ascidian Pyura praeputialis on a mid-intertidal rocky shore. - Mar. Ecol. Prog. Ser. 268: 119-130.

Crooks, J. A. 2002. Characterizing ecosystem-level consequences of biological invasions: the role of ecosystem engineers. - Oikos 97: 153-166.

Cuddington, K. and Hastings, A. 2004. Invasive engineers. - Ecol. Modell. 178: 335-347.

Cuddington, K. and Hastings, A. 2008. Balancing the engineerenvironment equation: the current legacy. - In: Cuddington, K. et al. (eds), Ecosystem engineers: plants to protists. Academic Press, pp. 253-273.

Cuddington, K. et al. 2009. Ecosystem engineers: feedback and population dynamics. - Am. Nat. 173: 488-498.

Dangerfield, J. et al. 1998. The mound-building termite Macrotermes michaelseni as an ecosystem engineer. - J. Trop. Ecol. 14: 507-520.

Darwin, C. 1881 . The formation of vegetable mould through the action of worms with some observations on their habits. - John Murray.

Dawkins, R. 2004. Extended phenotype - but not too extended. A reply to Laland, Turner and Jablonka. - Biol. Phil. 19: 377-396.

Flecker, A. S. 1997. Habitat modification by tropical fishes: environmental heterogeneity and the variability of interaction strength. - J. N. Am. Benthol. Soc. 16: 286-295.

Gilad, E. et al. 2004. Ecosystem engineers: from pattern formation to habitat creation. - Phys. Rev. Lett. 93: 1-4.

Gurney, W. S. C. and Lawton, J. H. 1996. The population dynamics of ecosystem engineers. - Oikos 76: 273-283.

Gutiérrez, J. L. and Jones, C. G. 2006. Physical ecosystem engineers as agents of biogeochemical heterogeneity. - Bioscience 56: $227-236$.

Gutiérrez, J. L. et al. 2003. Mollusks as ecosystem engineers: the role of shell production in aquatic habitats. - Oikos 101: 79-90.

Hastings, A. et al. 2007. Ecosystem engineering in space and time. - Ecol. Lett. 10: 153-164.

Hutchings, M. J. et al. 2000. The ecological consequences of environmental heterogeneity. - Blackwell.

Jones, C. G. and Gutiérrez, J. L. 2007. On the meaning, usage and purpose of the physical ecosystem engineering concept. - In: Cuddington, K. et al. (eds), Ecosystem engineers: plants to protists. Academic Press, pp. 3-24.

Jones, C. G. et al. 1994. Organisms as ecosystem engineers. - Oikos 69: 373-386.

Jones, C. G. et al. 1997. Positive and negative effects of organisms as physical ecosystem engineers. - Ecology 78: 1946-1957.

Johnston, C. A. and Naiman, R. J. 1990. Aquatic patch creation in relation to beaver population trends. - Ecology 71: $1617-1621$ 
Laland, K. N. et al. 1999. Evolutionary consequences of niche construction and their implications for ecology. - Proc. Natl Acad. Sci. USA 96: 10242-10247.

Lavelle, P. et al. 2007. Earthworms as key actors in self-organized soil systems. - In: Cuddington, K. et al. (eds), Ecosystem engineers: plants to protists. Academic Press, pp. 77-100.

Meadows, P. S. and Meadows, A. 1991. The environmental impact of burrowing animals and animal burrows. - Clarendon Press.

Moore, J. M. and Picker, M. D. 1991. Heuweltjies (earth mounds) in the Clanwilliam district, Cape Province, South Africa: 4000-year old termite nests. - Oecologia 86: 424-432.

Odling-Smee, J. F. et al. 2003. Niche construction: the neglected process in evolution. - Princeton Univ. Press.

Pickett, S. T. A. et al. 2000. Generation of heterogeneity by organisms: creation, maintenance, and transformation. - In: Hutchings, M. J. et al. (eds), The ecological consequences of environmental heterogeneity. Blackwell, pp. 33-52.

Reinhardt, L. et al. 2010. Dynamic interactions of life and its landscape: feedbacks at the interface of geomorphology and ecology. - Earth Surf. Proc. Land. 35: 78-101.

Saltelli, A. et al. 1999. A quantitative model-independent method for global sensitivity analysis of model output. - Technometrics 41: 39-56.

Shachak, M. et al. 2008. Woody species as landscape modulators and their effect on biodiversity patterns. - BioScience 58: 209-221.

Sharpe, F. A. 1984. Social foraging of the southeast Alaskan humpback whale, Meguptera novaeangliae. - PhD thesis, Univ Washington, Seattle.

Supplementary material (available online as Appendix O20283 at $<$ www.oikosoffice.lu.se/appendix $>$ ). Appendix A1-5.
Strayer, D. L. and Malcom, H. M. 2007. Shell decay rates of native and alien freshwater bivalves and implications for habitat engineering. - Freshwater Biol. 52: 1611-1617.

Van Hulzen, J. et al. 2007. Morphological variation and habitat modification are strongly correlated for the autogenic ecosystem engineer Spartina anglica (common cordgrass). - Estuar. Coast. 30: 3-11.

Volkenborn, N. et al. 2007. Effects of bioturbation and bioirrigation by lugworms (Arenicola marina) on physical and chemical sediment properties and implications for intertidal habitat succession. - Estuar. Coast. Shelf Sci. 74: 331-343.

Wilson, S. D. 2000. Heterogeneity, diversity and scale in plant communities. - In: Hutchings, M. J. et al. (eds), The ecological consequences of environmental heterogeneity. Blackwell, pp. 53-69.

Wright, J. P. 2009. Linking populations to landscapes: richness scenarios resulting from changes in the dynamics of an ecosystem engineer. - Ecology 90: 3418-3429.

Wright, J. P. and Jones, C. G. 2004. Predicting effects of ecosystem engineers on patch-scale species richness from primary productivity. - Ecology 85: 2071-2081.

Wright, J. P. et al. 2002. An ecosystem engineer, the beaver, increases species richness at the landscape scale. - Oecologia 132: 96-101.

Wright, J. P. et al. 2004. Patch dynamics in a landscape modified by ecosystem engineers. - Oikos 105: 336-348.

Wright, J. P. et al. 2006. Predictability of ecosystem engineering effects on species richness across environmental variability and spatial scales. - J. Ecol. 94: 815-824. 
Raynaud, X., Jones, C. G. and Barot, S. 2013. Ecosystem engineering, environmental decay and environmental states of landscapes. - Oikos 122: 591600.

\section{Appendix A1}

\section{Organismal movement within the landscape}

As pointed out in Model development, the model does not consider engineer movement within the landscape. We evaluated the degree to which movement qualitatively affected model outcomes using simulations based on a modified version of the general model (Eq. 6, 7). For each simulation run, at each time step, a fixed proportion of engineers, varying between 1 and $25 \%$ of the population in each environmental state, was designated as mobile and redistributed to other states based on two contrasting patterns that represented random or environmental state-dependent movement. Random movement was simulated by distributing all mobile organisms to all other states according to the proportions of each of those states; the greater the proportion of an environmental state in the landscape, the greater the number of organisms moving to that state. A simple representation of environmental state-dependent movement was simulated by transferring all mobile organisms to the least engineered state $\left(N_{l}\right)$, thereby decreasing the modification and maintenance of engineered states. Although movement had quantitative effects on landscape average environmental states and heterogeneities (e.g. changes in the variance of model outputs), the overall behaviour of the model was not altered by either kind of movement pattern, even when a relatively large proportion of organisms ( $25 \%$ for random movement; $10 \%$ for state-dependent movement) were moved at each time step (compare Fig. 4a and b with Fig. A3a and b and Fig. 3a with Fig. A3c). 

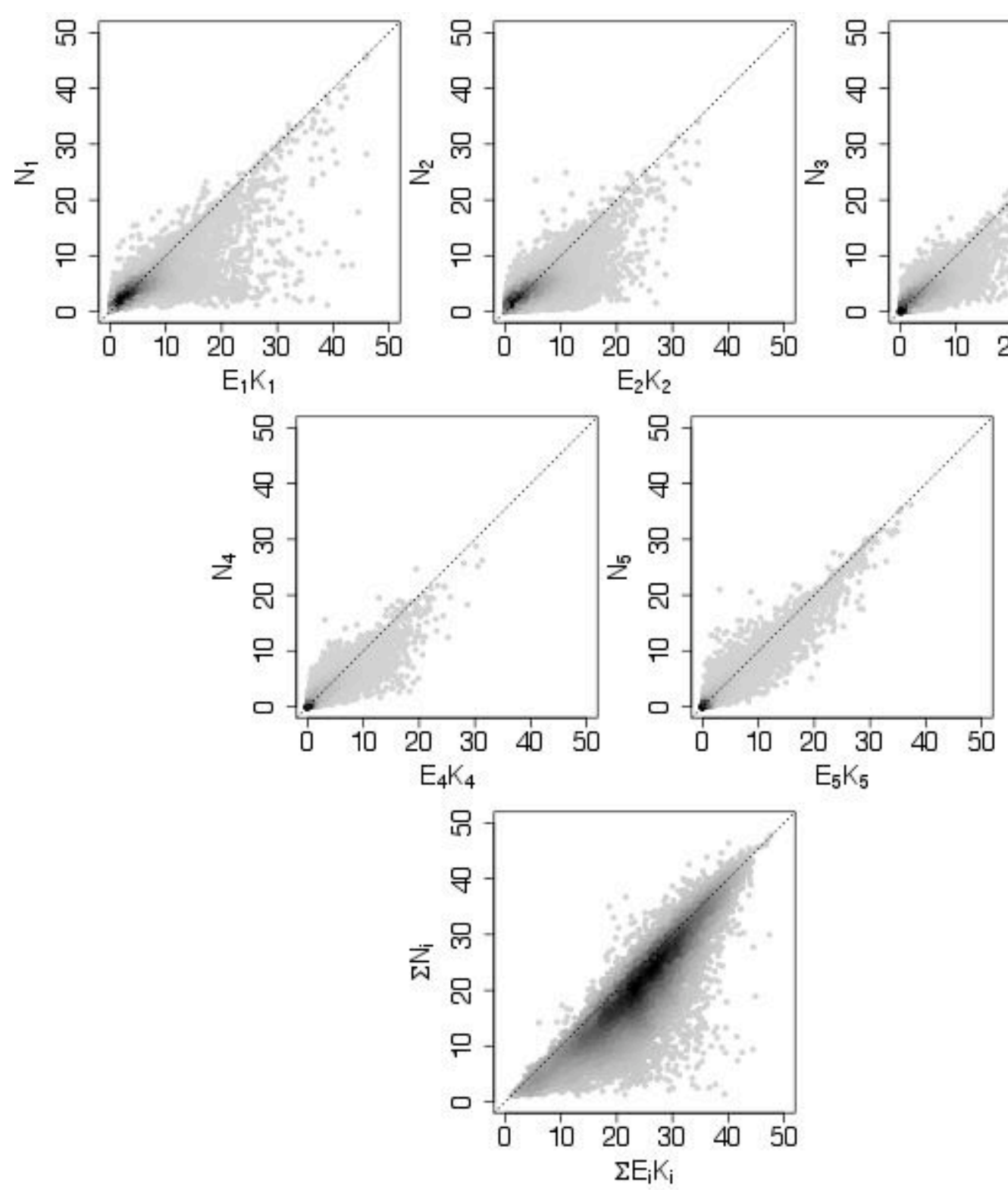

Figure A1. Relationships between realized $\left(\mathrm{N}_{\mathrm{i}}\right)$ and expected $\left(\mathrm{K}_{\mathrm{i}} \mathrm{E}_{\mathrm{i}}\right)$ engineer population sizes in each environmental state (subscript $1-5$ ), and the entire landscape $\left(\sum\right)$. In each panel, the dotted line is the 1:1 line. Each data point is the result of one simulation. Shading indicates data point density. Regressions between $\mathrm{N}_{\mathrm{i}}$ and $\mathrm{E}_{\mathrm{i}} \mathrm{K}_{\mathrm{i}}$ for states $\mathrm{i}=1, . ., 5$ have $\mathrm{R}^{2}$ values of $0.60,0.55,0.63,0.75,0.75$, respectively. The regression for the entire landscape has an $\mathrm{R}^{2}$ value of 0.73 . 

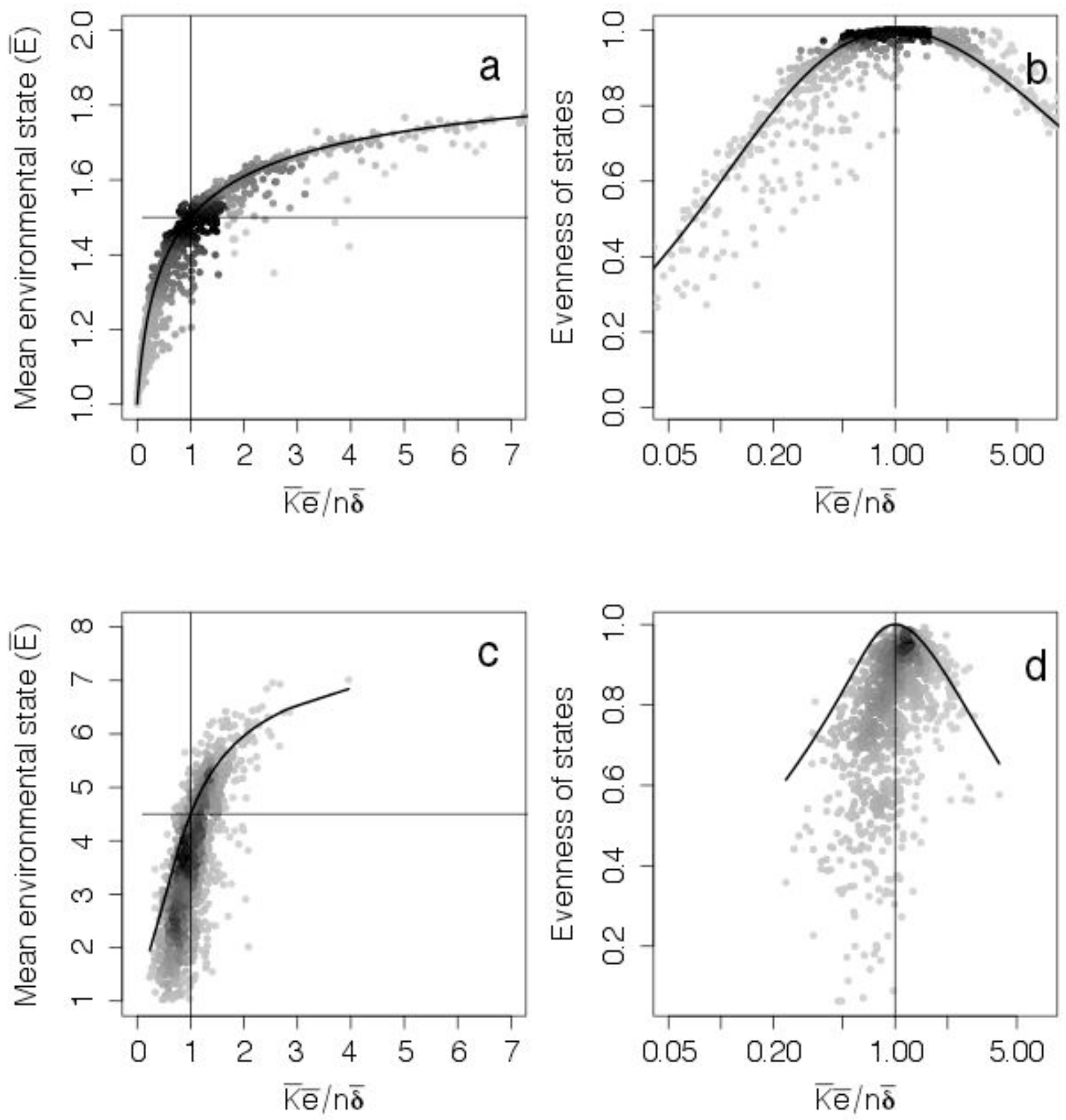

Figure A2. Relationships between mean environmental state of landscapes and NER (a, c) and evenness of environmental states and NER $(b, d)$ in the case of a model with 2 states $(a, b)$ and 8 states $(c, d)$. 

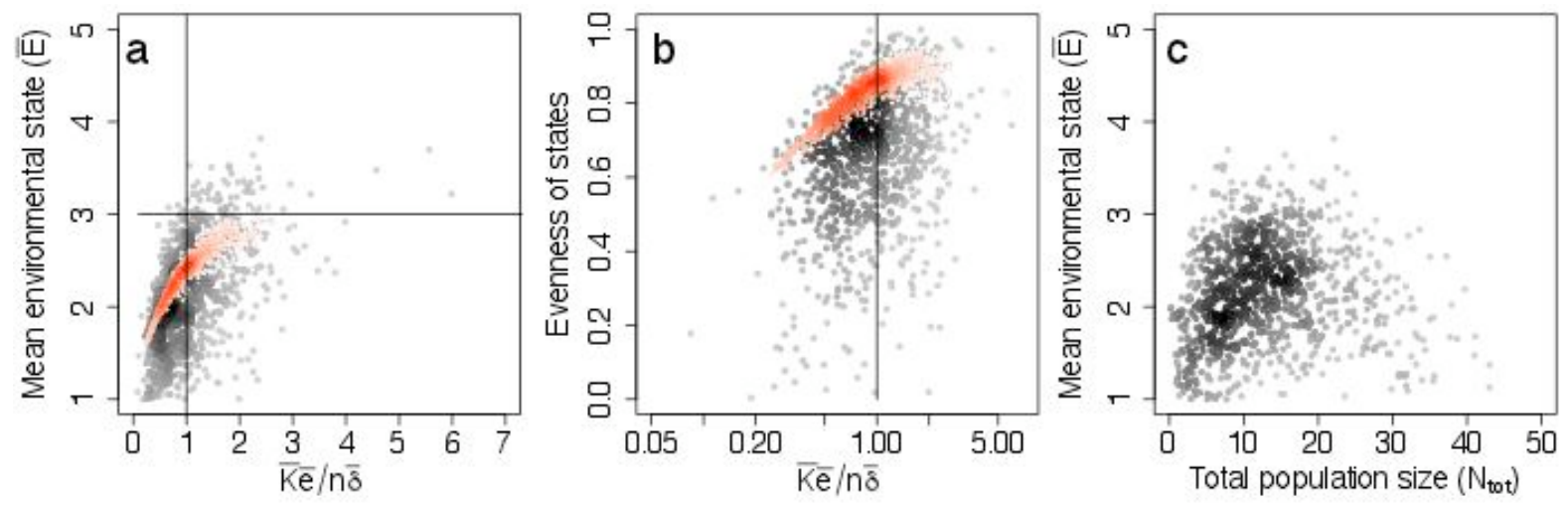

Figure A3. Relationships between (a) mean environmental state of landscapes and NER, (b) evenness of environmental states and NER and (c) mean environmental state of landscapes and total population size. For the case of 5 states, and where $25 \%$ of engineers of all states where moved to state 1 at each time step. Grey points correspond to simulations in the FR set whereas red points correspond to simulations in the AF set. 

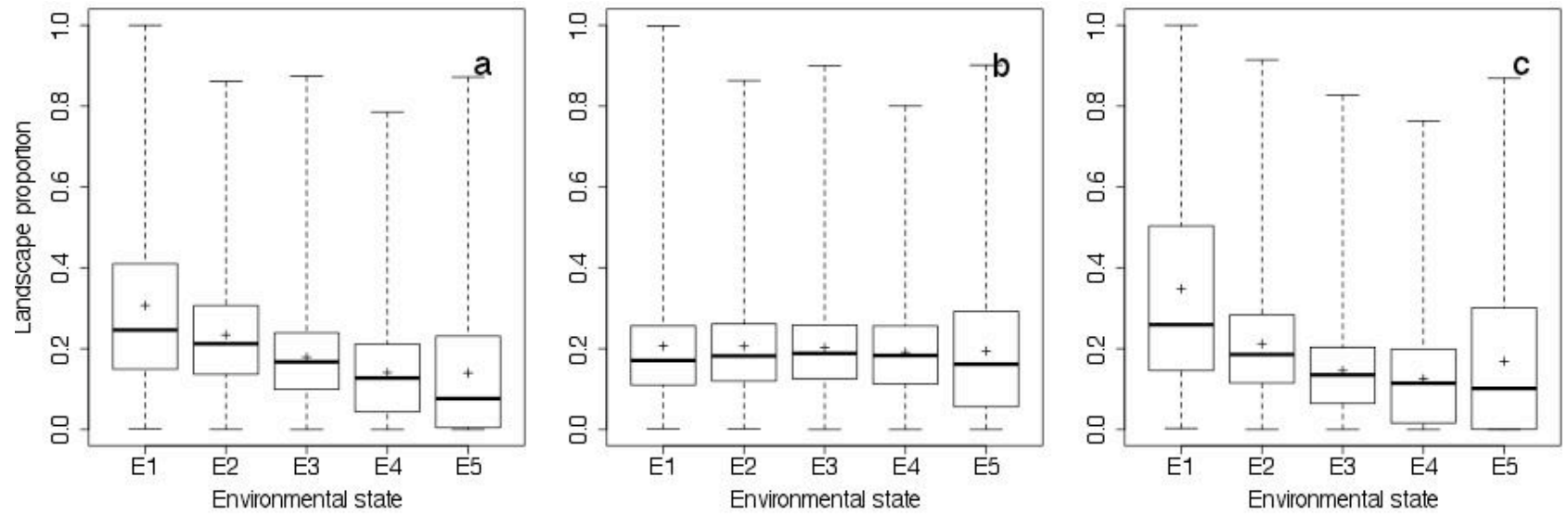

Figure A4. Proportions of the different environmental states across all simulations of the FR set (a), NF set (b) and PF set (c). 

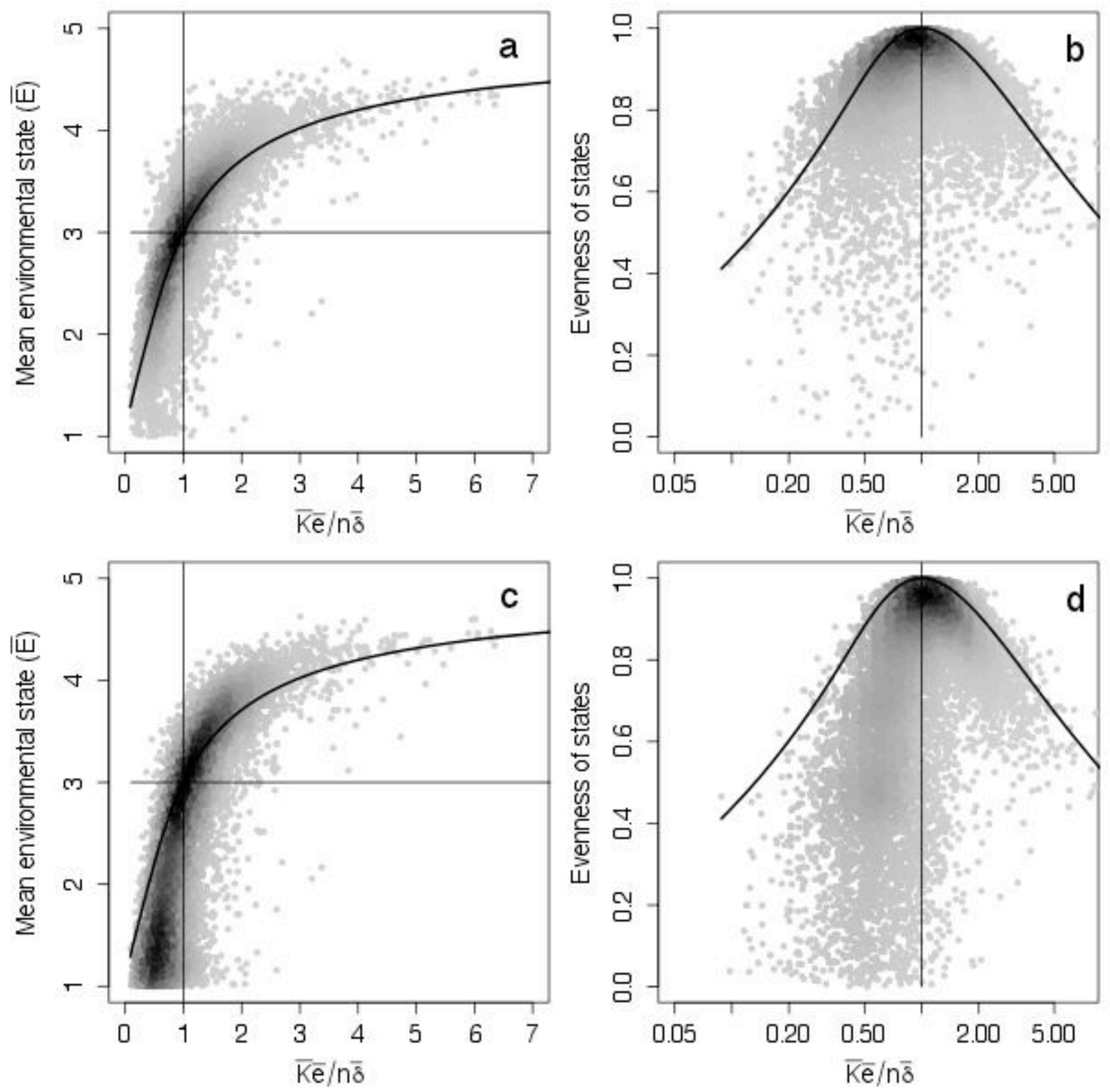

Figure A5. (a) and (c), relationship between mean environmental state of landscapes and NER for the case of 5 states. Thin vertical and horizontal lines show where NER $=1$ and the average state is equal to the intermediate state $=(\mathrm{n}+1) / 2$; (b) and (d), relationship between landscape environmental heterogeneities as evenness of environmental states, and NER. Note the logarithmic scale of the x-axis. Each data point is the result of one simulation. Shading indicates data point density. Panels (a) and (b), corresponds to the NF set, panels (c) and (d), to the PF set. The thick line on all graphs is the AR set. 